Reference: Parry R, Wade C A, Spearpoint M J. Implementing a glass fracture module in the BRANZFIRE zone model. Journal of Fire Protection Engineering, Vol. 13 No. 3, pp. 157183, 2003.

\title{
Implementing a Glass Fracture Module in the BRANZFIRE Zone Model
}

\author{
Ross PARRY $^{1}$, COLlEen A. WAdE ${ }^{2, *}$ AND MICHAEl SPEARPOINT ${ }^{3}$ \\ ${ }^{1}$ Holmes Fire and Safety, Wellington, New Zealand \\ ${ }^{2}$ BRANZ Limited, Wellington, New Zealand \\ ${ }^{3}$ University of Canterbury, Christchurch, New Zealand
}

\begin{abstract}
A glass fracture routine has been successfully implemented in the BRANZFIRE zone model, based largely on the heat transfer model developed by Sincaglia and Barnett and the fracture criterion of Pagni and Joshi. A second implementation of the model has been successfully created as a spreadsheet. The BRANZFIRE implementation has been compared against an existing glass fracture model, BREAK1 and against a series of experiments. The BRANZFIRE glass fracture model has been found to consistently predict marginally later fracture times than BREAK1 over a range of simulations. BRANZFIRE's fracture time prediction compared well with the experimental results. A Monte Carlo based sensitivity analysis found that fracture strength was the most important input variable, though this is largely attributable to the large standard deviation of the probability distribution used for this input.
\end{abstract}

KEY WORDS: fire modelling, verification and validation, glass fracture, Monte Carlo simulation, BRANZFIRE.

\section{INTRODUCTION}

This paper describes the implementation of a window glass fracture module within the BRANZFIRE fire zone model [1]. Such a module is fully interactive with the fire environment modelling, freeing the user from the need to manually specify vent opening times to account for window breakage. An appropriate model is one that is able to provide

\footnotetext{
*Author to whom correspondence should be addressed. Email: ColleenWade@branz.co.nz
} 
reasonable results without adding a large computational overhead to the BRANZFIRE software. The module has been verified against experimental data and existing glass fracture software. Further investigation into the sensitivity of the glass breaking time to various input parameters is of use to practitioners seeking to use the module for design work. This analysis provides guidance as to which input variables are of greatest significance. Some guidance on general window glass behaviour, including fracture and subsequent loss of integrity, is given based on examination of experimental reports and on insights gained through the modelling process. Further details regarding the research reported here can also be found elsewhere [2].

\section{LITERATURE REVIEW}

\section{Early Work}

Emmons [3] identified the need for research into the problem of glass breaking in fires. At that time, the only available research was from a research project carried out at Harvard which identified the mechanism through which glass breaks when heated.

Pagni [4] followed up on Emmons' suggestions and quantified the mechanics of the glass fracture problem, suggesting a failure criterion in terms of the glass temperature increase in the centre of a windowpane. Pagni and Joshi [5], Joshi and Pagni [6, 7, 8] and KeskiRahkonen [9, 10] later developed the theory required to model the heat transfer to a windowpane from a compartment fire.

\section{Existing Physical Models}

The glass fracture problem can be solved by separately considering the process of heat transfer from the fire and the hot gasses to the window glass and the process of the mechanical stress distribution and fracturing of the glass. Of these two processes, the heat transfer is the most difficult to model. The broader topic of glass breaking includes the post fracture behaviour of the window. A model for predicting the time for window glass to completely break and fall out of its framing has not been produced. Such a model would be important for accurately predicting the fracture of the second pane in a double or triple glazing system and for estimating the ability of glazing systems to resist impingement of external fires through the building envelope.

Cuzzillo and Pagni [11] summarise three possible models of the heat transfer problem. The simplest model treats the glass as a lumped mass and uses constant heat transfer coefficients, but this is inappropriate for application to rapid heating from fire. A more sophisticated approach is to treat the glass as a distributed mass that absorbs radiation through its thickness. 
The radiative heat transfer boundary conditions can be linearised for a relatively simple model or treated as non-linear for a more complete model. The temperature profile can be calculated in two dimensions, but a single dimensional model that calculates the temperature profile through the thickness of the glass is sufficient if a uniform heat flux is assumed.

Joshi and Pagni [7, 8] solve the heat transfer problem using a Laplace transform to produce integral equations that are then solved numerically using Newton-Raphson's method to find roots of the non-linear equations and the trapezoidal rule to approximate the integrals. This method requires evaluation of three integrals for each face of the windowpane at each time step. Each integral requires the evaluation of three very long kernel equations. Cuzzillo and Pagni [11] later extended this analysis to accommodate double-paned windows and exterior heating.

Keski-Rahkonen [9] has analysed the case of glass heated by radiation, as in the case of a fire burning in close proximity to a window, using linearised boundary conditions and assuming uniform through-thickness radiant energy absorption. The heat transfer problem is written as a Green function and integrated directly. An equation for the stress field generated by a given thermal field is then obtained by integrating the Airy's stress function. As stress waves travel much more quickly than thermal waves, the temperature field is treated as quasi-static. KeskiRahkonen [10] later extended this analysis from rectangular to circular panes.

Sincaglia and Barnett [12] developed a model for calculating glass window fracture for implementation in a zone type computer fire model. A numerical method for determining one dimensional heat transfer was adapted from a solution by Gardon [13]. A numerical solution method for solving the through-thickness temperature distribution, based on the standard explicit finite difference method is presented. The resulting temperature distribution can be averaged and compared against the glass fracture criterion from Pagni and Joshi [5]. Sincaglia and Barnett [12] pay particular attention to radiant energy absorption, transmission and emission within the glass and its functional dependence on wavelength.

\section{Existing Computer Models}

The most widely known glass-fracture model is BREAK1 [6], a DOS based program written in Fortran. The program is based on the heat transfer solution from Joshi and Pagni [5] and so models the glass as a distributed mass with through-thickness radiation absorption and nonlinear radiative boundary conditions. As well as specifying the dimensions and the thermophysical properties of the glass as constants, the user is required to enter the flame radiation flux history for windows close to the fire source, the hot layer temperature development, the 
heat transfer coefficient for the unexposed side (constant), the time-varying heat transfer coefficient for the fire-exposed side and the emissivity of the gas layer. The program then returns the temperature of both sides of the glass, the average non-dimensionalised temperature and the non-dimensionalised time, $\tau$, for each time step as well as the predicted time when the window fractures.

BREAK1 does not have the facility to run interactively with any zone modelling software. The user would therefore need to first run a zone model with the windows modelled as closed vents to obtain hot layer temperature and flame radiation flux histories (if available). The user can then run BREAK1 to determine the window fracture time, and finally run the zone model again, with the vent set to open when the window fractures. Naturally, the process could be repeated if there was more than one window in a compartment.

Cuzzillo and Pagni [11] extended BREAK1 to include the ability to model double-paned windows. The new program was rewritten using MathCAD and is named McBreak. Interpane radiant and convective heat transfer is modelled so that the windowpanes can break sequentially.

Sincaglia and Barnett [12] developed a glass window fracture model suitable for implementation in the WPI/Fire code. As the model is based on the explicit finite difference method, it is computationally less demanding than the solution used in BREAK1 and McBreak. The model also provides a better assessment of radiant heat transfer to the glass by separately considering the incident radiation in three wavelength bands. This model is also suitable for implementation into any zone-based computer fire model, including BRANZFIRE.

\section{Experimental Studies}

There have been a number of experimental investigations into fire induced fracture and breaking of framed and unframed, single- and double-glazed windows exposed to compartment fires, external fires and to direct heat fluxes.

Skelly, Roby and Beyler [14] tested framed and unframed glass exposed to compartment fires. They compared the temperature of a thermocouple on the interior surface of the glass at failure with the temperature predicted using Keski-Rahkonen's model. They found a discrepancy of $20^{\circ} \mathrm{C}$ between theory and experiment, which they attributed to direct radiative heating of the thermocouple. They also found that edge unprotected (unframed) glass could sustain approximately $100^{\circ} \mathrm{C}$ greater temperature increase before failure than framed glass. 
Joshi and Pagni [8] carried out a series of experiments to characterise three of the parameters required as inputs for their BREAK1 software model. They measured the fracture stress of 59 plate glass samples and obtained both a recommended value for design and a description of the statistical variation of fracture stresses. They also investigated the heat transfer coefficient for the exposed surface of the glass and the emissivity of the hot layer gases. These were not measured directly, but were estimated by fitting BREAK1 results to experimental data.

Experiments by Shields et al. [15] investigated the behaviour of large double glazed units exposed to full-scale office fire simulations. Wood cribs were used as fuel, gas temperatures, exposed and shaded glass temperatures and strains in the shaded edges were all reported.

Hassani et al. [16, 17] and Shields et al. [18, 19] have carried out a series of experimental investigations into glazing behaviour when exposed to fire. Their reports cover single and double-glazing tested in a half-scale compartment with a wood crib fire. The effect of nonuniform heating and the post-fracture behaviour of the glass are emphasized. Measurements include the gas temperature profiles within the compartment, shaded and exposed glass temperatures and thermally induced strains and stresses. Locations of fracture initiation and extent of bifurcation are also noted. Unfortunately, full details required for modelling the experiments as a means of verification are not given.

Harada et al [20] carried out an experimental study on glass cracking and fallout, using a gasfired radiant panel to expose the glass to imposed heat fluxes in the range $3-10 \mathrm{~kW} / \mathrm{m}^{2}$. They found that under intense heating (more than $9 \mathrm{~kW} / \mathrm{m}^{2}$ ) large pieces of glass fell out. With moderate heating the glass cracked but did not fall out.

More recently, Shields et al [21,22] have published the results of a series of experiments in which a single glazing assembly was exposed to fires of increasing severity in the centre, and in the corner of an enclosure ( $3.6 \mathrm{~m} \times 2.4 \mathrm{~m} \times 2.4 \mathrm{~m}$ ) instrumented and constructed to ISO room standards.

\section{Other Research}

Hassani et al. [23] review the importance of glass fracture to compartment fire behaviour, describe the mechanism of thermal fracture and discuss some issues associated with predicting glass fracture. A brief table-formatted literature summary is also provided. 
Mai and Jacob [24] conducted experiments on heat absorbing glasses, typically used as cladding in high-rise buildings, to assess thermal fracture induced by solar heating. The increase in fracture susceptibility caused by partial shading of glass panes is investigated and a method for calculating fracture strength based on the measured mirror radius of fracture surfaces is presented. As the fracture mechanism for solar heating is identical to that for fire heating, if only slower, much of this work is directly applicable to fire applications. A type of heat conducting sealant is proposed for use in susceptible windows to minimise the temperature difference between the exposed surface of the glass and the shaded edge.

Babrauskas [25] discusses glass breakage, concentrating on experimental studies but briefly mentioning theoretical studies. Computer models are not mentioned but a large number of experiments covering compartment fires and external radiation are discussed. The discussion is largely inconclusive, but may be a useful source of anecdotal evidence of glass fracture or non-fracture.

\section{PHYSICAL MODEL}

The physical model of heat transfer selected for implementation in the BRANZFIRE zone model generally follows the development by Sincaglia and Barnett [12] and is described below.

\section{Heat Transfer}

The two key heat transfer mechanisms from the hot upper gas layer to the window glass are convection at the interior surface and radiation absorption throughout the thickness of the glass. Heat is also transferred through the glass by conduction. Finally, heat is transferred from the hot glass to the external environment by means of convection at the exterior surface. Of these mechanisms, the radiation absorption through the thickness of the glass is the most difficult to model.

\section{Convection}

The convective heat transfer coefficient at the interior surface of the glass is dependent on the temperature of the fire environment and on the velocity of the hot gasses. A simplified correlation can be used to estimate this coefficient as follows [12]:

$$
h_{I}=h_{\min }+\left(h_{\max }-h_{\min }\right) \frac{\left(T_{U}-300\right)}{100}
$$


Where the values of $h_{\min }$ and $h_{\max }$ are 5 and $50 \mathrm{~W} / \mathrm{m}^{2} \mathrm{~K}$ respectively. The convective heat transfer coefficient at the exterior surface of the glass is not exposed to a large variation in temperature and is simply taken as a constant value of $h_{E}=10 \mathrm{~W} / \mathrm{m}^{2} \mathrm{~K}$.

\section{Radiation}

Window glass acts as a semitransparent medium, reflecting, transmitting and absorbing radiant energy. Only the energy that is absorbed contributes to the heating of the window glass. The rate of radiant energy transfer is a strong function of the temperature of the upper gas layer and is also proportional to the emissivity of the layer. Radiation may also be contributed from the flames and the bed of the fire, with the significance of this source depending on the distance between the flames and the window. The total irradiation from the environment, $G$ can be broken into three parts such that $G=\rho G+\alpha G+\tau G$ (see Figure 1).

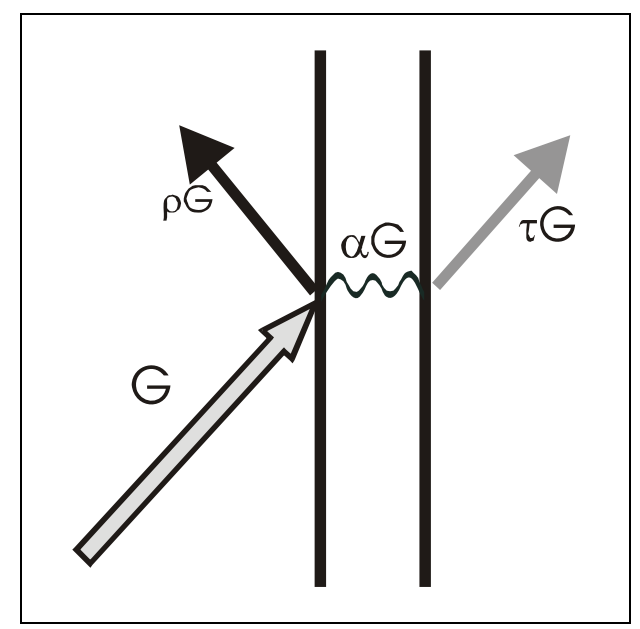

Figure 1 - Components of irradiation interacting with a semitransparent medium

The fractions $\rho, \tau$ and $\alpha$ are the reflectivity, transmissivity and absorptivity respectively. These ratios are functions of the angular distribution of the incident radiation, the distribution of wavelengths of the irradiance (hence the temperature of the upper layer) and the optical properties of the glass and air. To develop a model of radiant energy transfer, it is necessary to begin by isolating directional and then monochromatic radiant energy behaviour. To analyse directional radiation, the concept of intensity is required. Intensity is defined by Incropera and DeWitt [26] as the rate of radiant energy propagation in a particular direction, per unit area normal to the direction, per unit solid angle about the direction such that $I=\frac{d q}{d A \cos \theta d \omega}$. The angle $\theta$ is measured between the incident radiation and the normal 
to the receiving surface. Monochromatic intensity is a measure of the intensity of radiation for a single wavelength, $\lambda$, and is given the symbol $I_{\lambda}$.

The portion of radiant energy that will be reflected at the air-glass interface, thus never entering the glass, is dependent on the incident angle of the radiation and on the refractive indices of the air and the glass. The refracted angle is less than the incident angle in the case of radiant transfer from air to glass. The angles of incidence, $\theta_{1}$ and refraction, $\theta_{2}$ are related by $n_{\text {air }} \sin \theta_{1}=n_{\text {glass }} \sin \theta_{2}$.

The fraction of radiant energy that will be reflected at a particular incident angle is given by the Fresnel formula

$$
\rho\left(\theta_{1}\right)=\frac{1}{2} \frac{\sin ^{2}\left(\theta_{1}-\theta_{2}\right)}{\sin ^{2}\left(\theta_{1}+\theta_{2}\right)}\left[1+\frac{\cos ^{2}\left(\theta_{1}+\theta_{2}\right)}{\cos ^{2}\left(\theta_{1}-\theta_{2}\right)}\right]
$$

The radiation striking a glass surface from a hot upper layer is likely to have an almost uniform or 'diffuse' distribution of incident angle. Given that the radiation is diffuse, an average reflectivity can be calculated by averaging over the entire hemisphere of possible incident angles. Sincaglia and Barnett [12] give a double integral expression for the case of an air-glass interface, which, when evaluated gives the average reflectivity as 5.7\%. Therefore, the unreflected monochromatic intensity is given the symbol $I_{0 \lambda}$ and has a value of $0.943 \mathrm{I}_{\lambda}$.

The reduction in intensity of the radiation as it passes through the glass is a function of the absorption coefficient $\left(\gamma_{x}\right)$ for the wavelength under consideration and can be described as follows:

$$
\frac{d I_{\lambda}}{d x}=-\gamma_{\lambda} I_{\lambda}
$$

Where the $\mathrm{x}$ dimension is through the thickness of the glass. Separating variables and integrating this equation gives an expression for the monochromatic transmissivity:

$$
\tau_{\lambda}=e^{-\gamma_{\lambda} x}
$$

As it is the absorbed energy that contributes to the heating of the glass, the expression for the monochromatic unidirectional energy transfer is:

$$
\dot{q}_{\lambda}(x)=\left(1-e^{-\gamma_{\lambda} x}\right) I_{0 \lambda}
$$


Sincaglia and Barnett [12] show how this relationship is extended to allow for diffuse, fullspectrum radiation absorption leading to an expression for the monochromatic diffuse radiation energy transfer:

$$
\dot{q}_{\lambda}=\left(1-e^{-\gamma_{\lambda} \bar{l}}\right) G_{0 \lambda}
$$

where the non-directional analogue of intensity is the incident flux, $G$ and $G_{0 \lambda}=(1-\bar{\rho}) G_{\lambda}$. For an air/glass interface the average path length is evaluated as: $\bar{l}=1.077 \mathrm{~L}$

Finally, equation 6 must be extended to full spectrum radiation. The absorption coefficient of a material may vary as a function of the radiation wavelength and as a function of the temperature of the medium. For glass, at pre-fracture temperatures, the temperature dependence of the absorption coefficient is small and can be neglected. The wavelength dependence is much stronger, but can be represented as a stepped function, with constant absorption coefficient in three wavelength bands. Sincaglia and Barnett [12] use a stepped function for "typical window glass" shown in Figure 2.

The energy transfer can now be evaluated over three wavelength bands, given that the fraction of the radiant energy from the hot layer within each band is known. The fractional energy function, $f_{0 \rightarrow \lambda}$, can be used to calculate the fraction of energy contained in the band from $\lambda=0$ to $\lambda=\lambda_{1}$ as follows:

$$
f_{0 \rightarrow \lambda T}=1-\frac{15}{\pi^{4}} \int_{0}^{\xi} \frac{\xi^{3} d \xi}{e^{\xi}-1} d \xi, \quad \text { where } \xi_{\lambda T}=\frac{C_{2}}{\lambda T}
$$

Where $T$ is the temperature of the radiation source (the upper layer) and $C_{2}$ is a constant equal to $14387.69 \mu \mathrm{mK}$. A solution to this equation can be obtained using the following converging series [27]:

$$
f_{0 \rightarrow \lambda T}=\frac{15}{\pi^{4}} \sum_{n=1}^{\infty}\left[\frac{e^{-n \xi}}{n}\left(\xi^{3}+\frac{3 \xi^{2}}{n^{2}}+\frac{6 \xi}{n^{2}}+\frac{6}{n^{3}}\right)\right]
$$




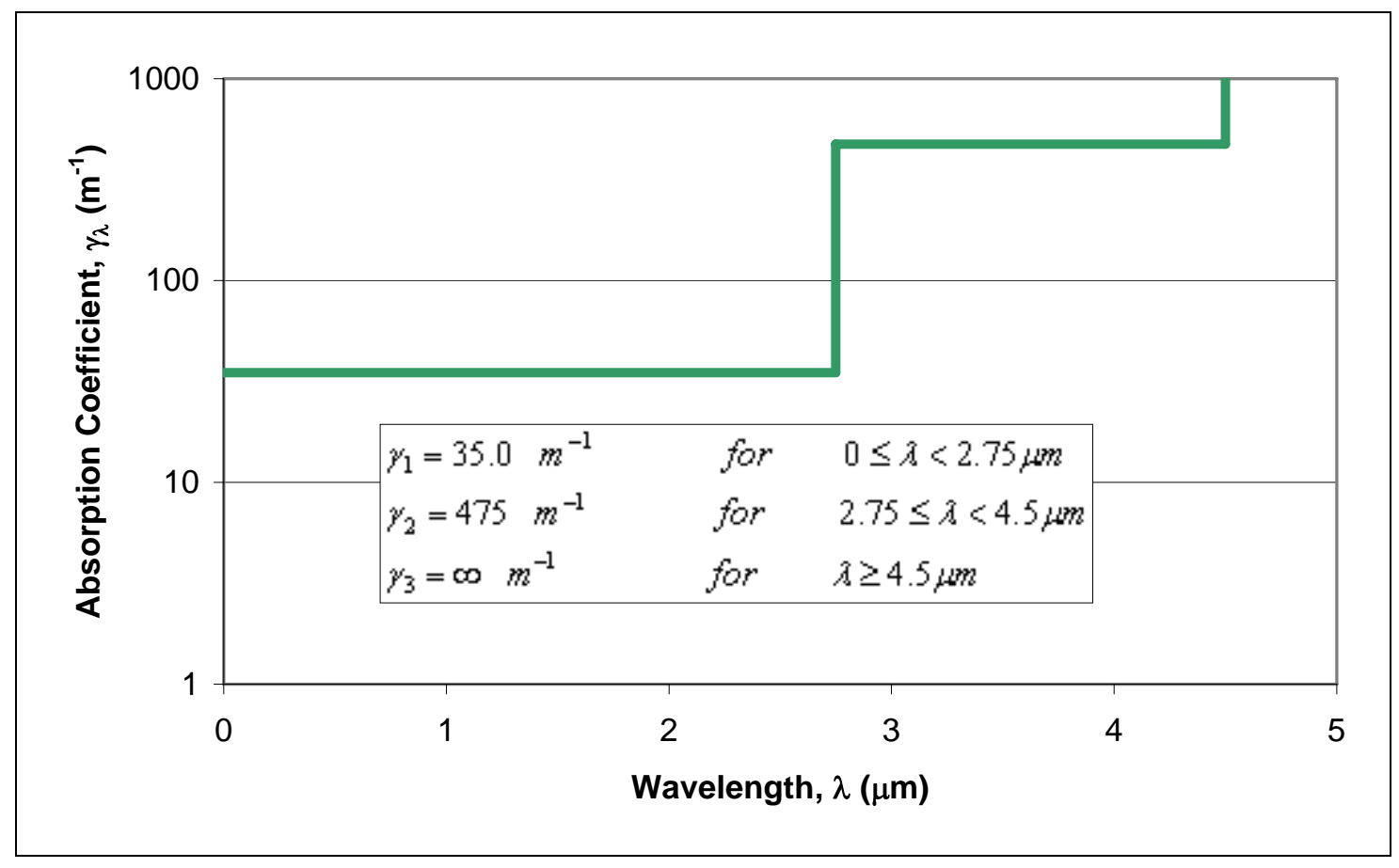

Figure 2 - Stepped absorption coefficient approximation for typical window glass

The development of the model for radiant heat transfer from a radiating source (the hot upper layer) is now complete and can be summarised as follows:

$$
\begin{aligned}
& \dot{q}=\dot{q}_{1}+\dot{q}_{2}+\dot{q}_{3} \\
& \dot{q}_{i}=(1-\bar{\rho})\left(1-e^{-\gamma_{i} \bar{l}}\right) f_{\lambda_{i-1} \rightarrow \lambda_{i}} \varepsilon_{U} \sigma T_{U}^{4} \\
& f_{\lambda_{i-1} \rightarrow \lambda_{i}}=f_{0 \rightarrow \lambda_{i}}-f_{0 \rightarrow \lambda_{i-1}}
\end{aligned}
$$

The heated glass will also emit radiation, some of which will be reabsorbed within the pane. This will result in some redistribution of energy within the glass. Sincaglia and Barnett [12] allow for this redistribution by using Endry and Turzik's apparent conductivity correlation where $k^{\prime}(T)=0.7222+0.001583 T$.

\section{Temperature Distribution}

The temperature distribution is calculated through the thickness of the glass only. This onedimensional temperature distribution is averaged and used to assess whether fracture has occurred. Nodes are selected at the interior and exterior surfaces of the glass and spaced evenly through the thickness. The temperature at each node is calculated at each time step using transient explicit finite difference formulae that can be written from consideration of the energy balance for each node. Using the convention that heat flows into the node under 
consideration, the energy balance for the interior node can be written in finite-difference formulation as follows:

$$
h_{I}\left(T_{U}-T_{0}^{P}\right)+\frac{k^{\prime}}{\Delta x}\left(T_{1}^{P}-T_{0}^{P}\right)+\dot{q}_{\text {rad }}=\rho c_{p} \frac{\Delta x}{2} \frac{T_{0}^{P+1}-T_{0}^{P}}{\Delta t}
$$

Where the superscript indicates the time step, with $p$ being the current time step and $p+1$ being the next time step. $k^{\prime}$ is the apparent conductivity as defined in the previous section. The term $\dot{q}_{\text {rad }}$ indicates any radiative heat transfer from the hot upper layer to the volume surrounding the node under consideration. The effect of nodal spacing on the magnitude of the radiative heat transfer is incorporated into the evaluation of $\dot{q}_{\text {rad }}$. The explicit finite difference formula for the temperature of the interior surface node is obtained by solving for $T_{0}^{P+1}$ :

$$
T_{0}^{P+1}=T_{0}^{P}+\frac{2 \Delta t}{c_{p} \rho \Delta x}\left[h_{I}\left(T_{U}-T_{0}^{P}\right)-\frac{k^{\prime}}{\Delta x}\left(T_{1}^{P}-T_{0}^{P}\right)+\dot{q}_{\text {rad }}\right] \quad \text { Interior node }
$$

For the interior node, $\dot{q}_{\text {rad }}$ equals $\dot{q}_{1}+\dot{q}_{2}+\dot{q}_{3}$, all evaluated with $\bar{l}=1.077 \Delta x / 2$.

Similar development for the internal and exterior nodes yields the following formulae:

$$
\begin{array}{rr}
T_{m}^{P+1}=T_{m}^{P}+\frac{\Delta t}{\rho c_{p} \Delta x}\left[\frac{k^{\prime}}{\Delta x}\left(T_{m-1}^{P}+T_{m+1}^{P}-2 T_{m}^{P}\right)+\dot{q}_{\text {rad }}\right] & \text { Internal nodes } \\
T_{N}^{P+1}=T_{N}^{P}+\frac{2 \Delta t}{\rho c_{p} \Delta x}\left[h_{E}\left(T_{\infty}-T_{N}^{P}\right)+\frac{k^{\prime}}{\Delta x}\left(T_{N-1}^{P}-T_{N}^{P}\right)+\dot{q}_{r a d}\right] & \text { Exterior node }
\end{array}
$$

For the internal nodes, $\dot{q}_{\text {rad }}$ equals $\dot{q}_{1}+\dot{q}_{2}$, evaluated with $\bar{l}=1.077 \Delta x$. The quantity $\dot{q}_{3}$ is not included because this represents radiant energy in the spectral band for which glass is opaque. Absorption of the energy $\dot{q}_{3}$ is thus essentially a surface phenomenon and therefore only affects the temperature of the interior node.

For the exterior node, $\dot{q}_{\text {rad }}$ equals $\dot{q}_{1}+\dot{q}_{2}+\dot{q}_{\infty}$, evaluated with $\bar{l}=1.077 \Delta x / 2$. The quantity $\dot{q}_{\infty}$ is surface-absorbed radiant energy from the external environment. This radiant energy is treated as surface absorbed because the ambient temperature is considered low enough that most of the energy is within the spectral range for which glass is opaque. 
Explicit finite-difference schemes are not unconditionally stable. The stability limit for the time step can be found by setting the coefficients of the previous time step's nodal temperature greater than, or equal to zero. In this case, the interior node provides the dominant stability criterion and is evaluated as follows:

$$
0 \leq 1-\frac{2 \Delta t}{\rho c_{p} \Delta x}\left(h_{I}+\frac{k^{\prime}}{\Delta x}\right) \quad \Rightarrow \Delta t \leq \frac{\rho c_{p}(\Delta x)^{2}}{2\left(h_{I} \Delta x+k^{\prime}\right)}
$$

This differs from the stability criterion given by Sincaglia and Barnett [12], which was found to under-predict the maximum stable time step. The revised stability criterion has proven more reliable. Selecting a larger number of nodes will decrease both the spacing between nodes and the upper limit on the time step. This means that selecting a large number of nodes is computationally demanding both in terms of the number of temperatures to be calculated through the thickness of the glass and in terms of the number of times the calculations must be performed. The trade-off is in the accuracy of the calculations. Choosing a large number of nodes will provide a more accurate result.

\section{Fracture}

The fracture of the windowpane is generally initiated at an edge defect. Defects are introduced during manufacture and when the glass is cut to size and act as sites of stress concentration. Fracture occurs when thermally induced tensile stresses in the shaded edge reach the tensile defect strength. A simple relationship is used to assess when the temperature increase in the exposed portion of the pane is sufficient to induce a predetermined failure stress in the shaded edge. This relationship is as follows:

$$
\overline{\Delta T}=\frac{1}{N} \sum_{i}^{N} T_{i}-T_{0} \geq\left(1+\frac{s}{H}\right) \frac{\sigma_{f}}{E \beta}
$$

Where $T_{i}$ is the temperature at the $\mathrm{i}^{\text {th }}$ node, $\mathrm{N}$ is the number of nodes, $T_{0}$ is the initial temperature, $\mathrm{s}$ is the shaded length, $H$ is the half-width of the window, $\mathrm{E}$ is the Young's modulus of the glass, $\beta$ is the coefficient of thermal expansion and $\sigma_{f}$ is the fracture strength. This relationship was developed by Pagni and Joshi [5]. This relationship may be altered slightly if the framing is so tight as to restrict expansion or to impose edge stresses prior to heating. Half width and shaded length are illustrated in Figure 3. 


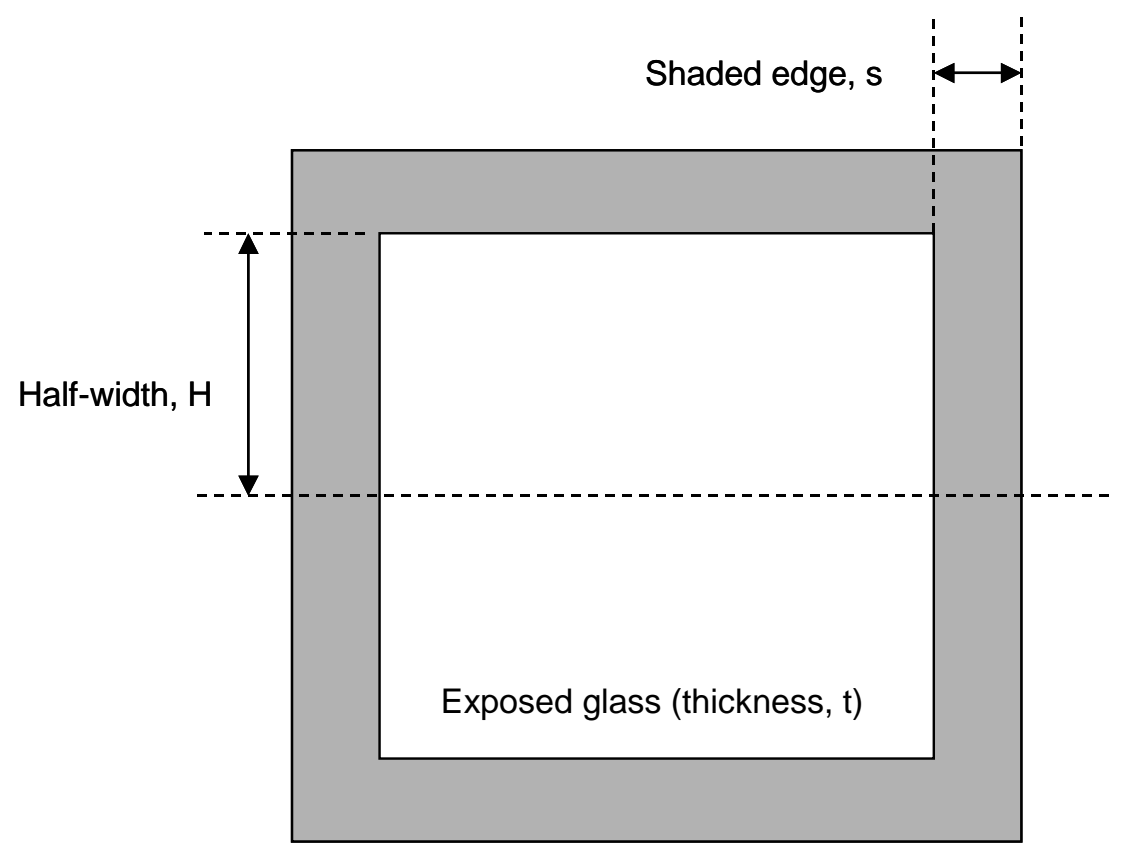

Figure 3 - Window dimensions

\section{Limitations of the Physical Model}

There are several limitations to the glass fracture model as presented above:

- The fracture criterion assumes full immersion of the glass in the hot layer and thus uniform heating of the exposed surface.

- The fracture criterion application is limited to the case of a rectangular window. Circular or oval windows cannot be modelled.

- Only planar windows can be assessed.

- The model is not applicable to thermal shock modelling such as might be encountered in explosions or during sprinkler activation.

- The model is suited to heating from internal compartment fires only and cannot be used to assess fracture caused by external fires such as wildfires or flames from lower floors.

- Double-glazed windows are capable of maintaining their integrity for much longer than single panes. Significant heating of the second pane does not occur until the first pane has begun to fall out.

- There is no model available for predicting glass fall-out time. The model predicts time to first fracture only. Some experiments have shown that windows may take a very long time to fall out following first fracture.

- The joint action of thermal stresses and out-of-plane loading is not considered. Outof-plane loading may occur due to pressure on the pane from sources such as wind or 
explosions. Thermal and pressure fractures are unlikely to affect each other, but pressure loading may affect fall-out times following first fracture.

In addition, the fracture temperature criterion was developed for the case where the window has uniform exposure to the fire environment. Experimental work by Hassani et al [16] suggests that the stress fields are significantly different for windows exposed to a two-layer fire environment over their height. The increasing use of large windows in modern buildings means that windows will often be exposed to a hot upper layer and a cooler lower layer during the development of a fire. More work is needed to investigate the effect of nonuniform heating on the fracture temperature criterion.

\section{VERIFICATION}

Results obtained from the glass fracture model were compared with the BREAK1 fracture times, and with times from reported glass fracture experiments.

\section{BREAK1 Comparison}

The BREAK1 code uses a significantly different heat transfer model to that implemented in BRANZFIRE. BREAK1 is based on the model developed by Pagni and Joshi [5], Joshi and Pagni [7] and uses numerical methods to solve an analytical physical description of the heat transfer to the glass. BREAK1 uses a single radiative decay length value (the inverse of the absorption coefficient) for the entire radiation spectrum.

Comparisons were made without using flame flux in either program. Simulations were run with a single window in a standard ISO room, with a range of fires and glass thicknesses. The BRANZFIRE predicted values for compartment gas temperature and layer emissivity were used as input to BREAK1 since the latter is not a compartment model. The scenarios are summarised in Table 1 and the results are presented in Figure 4.

The results show excellent agreement between BRANZFIRE (version 2002.6) and BREAK1, particularly for the 3 and $6 \mathrm{~mm}$ glass thicknesses. For the $12 \mathrm{~mm}$ thick glass, agreement was also reasonable but with BRANZFIRE fracture times generally slightly quicker than those predicted by BREAK1. 


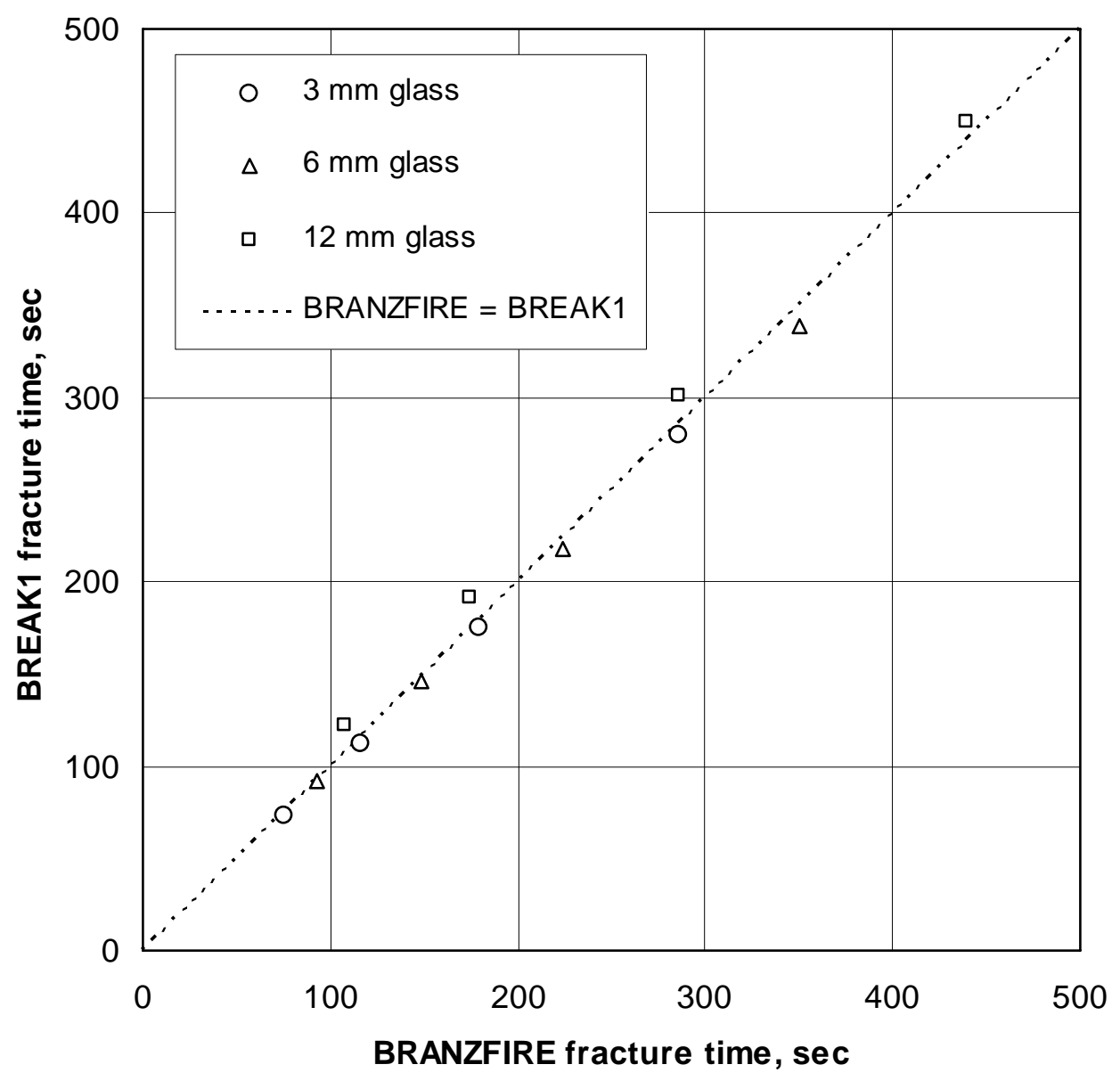

Figure 4 - BREAK1 versus BRANZFIRE fracture time comparison results ISO Room, $t$-squared fire, no flame flux heating

Table 1 - BREAK1 versus BRANZFIRE comparison simulation scenarios

\begin{tabular}{|l|l|}
\hline \multicolumn{1}{|c|}{ Property } & \multicolumn{1}{c|}{ Value(s) } \\
\hline Room size & $2.4 \times 3.6 \times 2.4 \mathrm{~m}$ high \\
\hline Opening & $0.8 \times 2.0 \mathrm{~m}$ high \\
\hline Window geometry & $0.8 \times 0.8 \mathrm{~m}$, sill at $1.0 \mathrm{~m}$ \\
\hline Glass geometry & $\begin{array}{l}3 \mathrm{~mm} \text { thick, } 12 \mathrm{~mm} \text { shaded edge } \\
6 \mathrm{~mm} \text { thick, } 15 \mathrm{~mm} \text { shaded edge } \\
12 \mathrm{~mm} \text { thick, } 25 \mathrm{~mm} \text { shaded edge }\end{array}$ \\
\hline Glass properties & $\mathrm{k}=0.76 \mathrm{~W} / \mathrm{mK}, \alpha=3.6 \mathrm{E}-07, \mathrm{E}=72 \mathrm{GPa}$, \\
& $\sigma_{\mathrm{f}}=47 \mathrm{MPa}, \beta=.95 \mathrm{E}-05 \mathrm{~K}^{-1}$ \\
\hline Fires & $\begin{array}{l}\text { Slow, medium, fast and ultra-fast } \\
\text { (t-squared growth) }\end{array}$ \\
\hline
\end{tabular}




\section{Experimental Comparison}

\section{Experiments of Skelly, Roby and Beyler}

A set of completely documented compartment fire experiments against which to compare the glass fracture model are those of Skelly, Roby and Beyler [14]. The experimental setup consists of a 1.0 m x 1.2 m x 1.5 m high compartment lined with Kaowool ceramic fibre board. The compartment has open exhaust and inlet vents and a closed window. The inlet vent is in a plenum at the base of the fire compartment. A series of tests were conducted with pans of hexane ignited in the centre of the compartment. BRANZFIRE (version 2002.6) [1] has been used to simulate the fire development and glass fracture for a $20 \mathrm{~cm}$ x $20 \mathrm{~cm}$ pan fire. The WPI/Fire model has also been verified against this experiment [12].

The peak release rate of the fire was calculated using pool fire correlations and published properties for the hexane fuel. The mass loss rate equation for a pool fire is given by Babrauskas [28] as:

$$
\dot{m}^{\prime \prime}=\dot{m}_{\infty}^{\prime \prime}\left(1-e^{-k \beta D}\right)
$$

Where $\dot{m}_{\infty}^{\prime \prime}$ is the mass loss rate of an infinitely large pool, $D$ is the actual pool diameter and $k \beta$ is a physical property of the fuel. For hexane the relevant properties are $0.074 \mathrm{~kg} / \mathrm{m}^{2} \mathrm{~s}$ and $1.9 \mathrm{~m}^{-1}$ for $\dot{m}_{\infty}^{\prime \prime}$ and $k \beta$ respectively. The equivalent diameter of a $20 \mathrm{~cm} \mathrm{x} 20 \mathrm{~cm}$ pan is $0.23 \mathrm{~m}$ giving $\dot{m}^{\prime \prime}=0.026 \mathrm{~kg} / \mathrm{m}^{2} \mathrm{~s}$.

With a heat of combustion of $44.7 \mathrm{~kJ} / \mathrm{g}$ and combustion efficiency $\chi=0.92$ for hexane [29], the estimated heat release rate is $43 \mathrm{~kW}$. Sincaglia and Barnett [12] assumed a combustion efficiency $\chi=0.65$ suggesting an estimated heat release rate of only $30 \mathrm{~kW}$. Since the actual combustion efficiency (and heat output) applicable to the experiments is not known, and since the predicted fracture times are very sensitive to the heat output from the fire, results from BRANZFIRE were obtained using both values. Carbon dioxide, water vapour and soot yields for hexane based on well-ventilated conditions were sourced from Tewarson [29]. A radiant loss fraction from the fire plume of 0.35 was assumed for all the BRANZFIRE simulations.

The fracture time in BRANZFIRE was 103 seconds taking the lower value for combustion efficiency. This is compared with the experimental results, with WPI/Fire and with BREAK1 in Table 2. 
Table 2 - Comparison of experimental and various calculated fracture times

\begin{tabular}{|l|l|l|}
\hline \multicolumn{3}{|c|}{ Fuel - 20 x 20 cm square pan of hexane } \\
\hline Source & Fracture Time(s) & Comments \\
\hline BRANZFIRE & 73 seconds & $(\chi=0.92)$ \\
\hline BRANZFIRE & 103 seconds & $(\chi=0.65)$ \\
\hline WPI/ Fire & 110 seconds & $\begin{array}{l}\text { Sincaglia and Barnett [12] } \\
(\chi=0.65)\end{array}$ \\
\hline BREAK1 & 96 seconds & Joshi and Pagni [8] \\
\hline Experiment & $\begin{array}{l}109,100,112 \text { seconds } \\
(107 \text { average })\end{array}$ & $\begin{array}{l}\text { Experiment repeated three } \\
\text { times. Tests 4, 5 and 6. Skelly, } \\
\text { Roby and Beyler [14] }\end{array}$ \\
\hline
\end{tabular}

The experiment was repeated three times by Skelly, Roby and Beyler, with a range of fracture times recorded. The BRANZFIRE prediction was within the range measured experimentally. The fracture time for BREAK1 is less than BRANZFIRE's but the inputs to BREAK1 were not fully known, and in particular there was manipulation of the gas emissivity and convective heat transfer coefficients to obtain good agreement for the breaking times [8]. The calculated fracture time from WPI/Fire was slightly higher than the BRANZFIRE prediction.

It is also noted that Skelly, Roby and Beyler [14] present a gas temperature history for the compartment fire that differs significantly from that predicted by BRANZFIRE either with or without the plenum. At fracture time, the gas temperature is shown as almost $400^{\circ} \mathrm{C}$, compared to only $164^{\circ} \mathrm{C}$ predicted by BRANZFIRE. Sincaglia and Barnett do not show the gas layer temperature profile that they have obtained. Higher than actual temperatures may have been read by the gas thermocouples because of radiative heating. A comparison of the interior surface temperature of the glass obtained from WPI/Fire, BRANZFIRE and the Skelly et al experiments is shown in Figure 5.

WPI/Fire and BRANZFIRE have similar temperature profile predictions. This suggests that the gas layer temperature given by Skelly, Roby and Beyler may not be accurate. Sincaglia and Barnett do not present the gas temperature profile that was calculated by WPI/Fire, so it is not possible to make a direct comparison with BRANZFIRE's calculations. Further comparisons using a $30 \times 20 \mathrm{~cm}$ and $20 \mathrm{~cm}$ diameter pans are shown in Table 3 and Table 4. In both cases the fracture times predicted by BRANZFIRE span the times experimentally determined by Skelly, Roby and Beyler, and suggest that perhaps the true combustion efficiency may lie somewhere between 0.65 and 0.92 for the hexane pans. The effect that the magnitude of the heat release rate has on the predicted fracture times is also significant. 


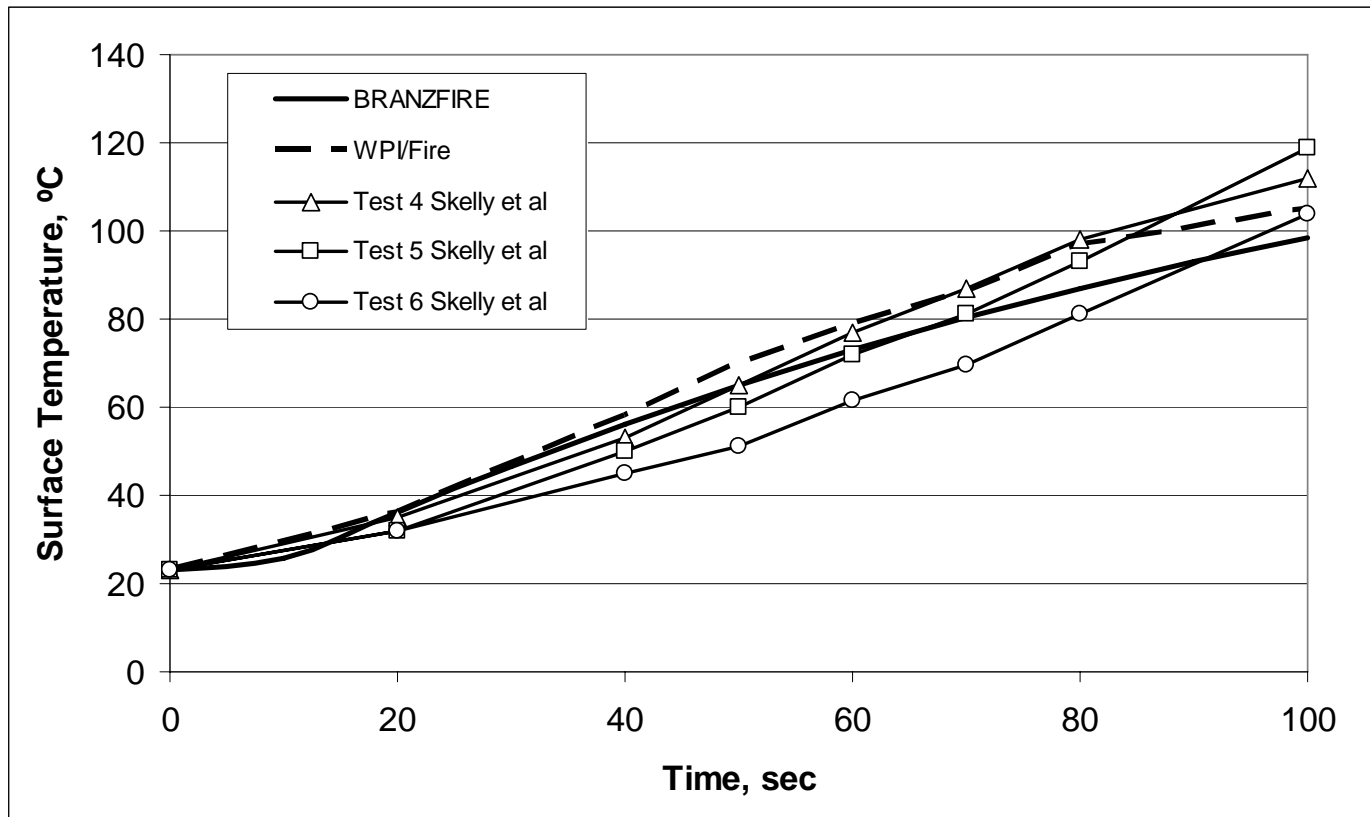

Figure 5 - Interior surface temperatures - BRANZFIRE, WPI/Fire and Experiments for $20 \times 20 \mathrm{~cm}$ pan of hexane

Table 3 - Comparison of fracture times $30 \times 20 \mathrm{~cm}$ pan of hexane

\begin{tabular}{|c|c|c|}
\hline \multicolumn{3}{|c|}{ Fuel - 30 x $20 \mathrm{~cm}$ pan of hexane } \\
\hline Source & Fracture Time(s) & Comments \\
\hline BRANZFIRE & 47 seconds & $(x=0.92)$ \\
\hline BRANZFIRE & 62 seconds & $(\chi=0.65)$ \\
\hline WPI/ Fire & 55 seconds & $\begin{array}{l}\text { Sincaglia and Barnett [12] } \\
(\chi=0.65)\end{array}$ \\
\hline BREAK1 & 54 seconds & J oshi and Pagni [8] \\
\hline Experiment & $\begin{array}{l}55,48,56 \text { seconds } \\
\text { (53 average) }\end{array}$ & $\begin{array}{l}\text { Experiment repeated three } \\
\text { times. Tests } 1 \text {, } 2 \text { and } 3 \text {. Skelly, } \\
\text { Roby and Beyler [14] }\end{array}$ \\
\hline
\end{tabular}

Table 4 - Comparison of fracture times for $20 \mathrm{~cm}$ diameter pan of hexane

\begin{tabular}{|c|c|c|}
\hline \multicolumn{3}{|c|}{ Fuel - $20 \mathrm{~cm}$ diameter pan of hexane } \\
\hline Source & Fracture Time(s) & Comments \\
\hline BRANZFIRE & 104 seconds & $(\chi=0.92)$ \\
\hline BRANZFIRE & 156 seconds & $(\chi=0.65)$ \\
\hline WPI/Fire & 150 seconds & $\begin{array}{l}\text { Sincaglia and Barnett [12] } \\
(\chi=0.65)\end{array}$ \\
\hline Experiment & 127,132 seconds & $\begin{array}{l}\text { Experiment repeated twice. } \\
\text { Tests } 7 \text { and } 8 \text {. Skelly, Roby and } \\
\text { Beyler [14] }\end{array}$ \\
\hline
\end{tabular}




\section{Experiments of Shields, Silcock and Flood}

Pan fires of varying size burning mineralised methylated spirits were located in the corner and in the centre of a vented compartment 3.6 × 2.4 x $2.4 \mathrm{~m}$ as described by Shields, Silcock and Flood [21, 22]. The pans were elevated $300 \mathrm{~mm}$ above floor level. There was a doorway vent $0.4 \mathrm{~m}$ wide $\times 2.0 \mathrm{~m}$ high, and a glazed window assembly comprising three panes. Pane 1 measured $0.844 \mathrm{~m} \times 0.844 \mathrm{~m}$ with the sill at a height of $1.06 \mathrm{~m}$. Pane 2 measured $0.844 \mathrm{~m} \mathrm{x}$ $0.844 \mathrm{~m}$ with the sill at floor level. Pane 3 measured $0.844 \mathrm{~m}$ wide $\mathrm{x} 1895 \mathrm{~m}$ high with the sill at floor level. In all cases the glazing was $6 \mathrm{~mm}$ thick with a $20 \mathrm{~mm}$ shaded edge and with properties $\left(\mathrm{k}=0.937 \mathrm{~W} / \mathrm{mK}, \alpha=4.2 \mathrm{E}-07, \mathrm{E}=72 \mathrm{GPa}, \quad \beta=.83 \mathrm{E}-05 \mathrm{~K}^{-1}\right)$ for soda-lime-silica float glass taken from Pilkington technical literature [30].

Table 5 - Comparison with FireSERT Compartment Fire Tests

\begin{tabular}{|c|c|c|c|c|c|c|}
\hline & $\begin{array}{l}\text { Pane 1- } \\
(0.844\end{array}$ & $\begin{array}{l}\text { ill } 1.06 \mathrm{~m} \\
.844 \mathrm{~m})\end{array}$ & $\begin{array}{l}\text { Pane } 2 \\
(0.844\end{array}$ & $\begin{array}{l}\text { sill } 0 \mathrm{~m} \\
0.844 \mathrm{~m} \text { ) }\end{array}$ & $\begin{array}{l}\text { Pane } 3 \text { - } \\
(0.844 \text { x }\end{array}$ & $\begin{array}{l}\text { ill } 0 \mathrm{~m} \\
.895 \mathrm{~m})\end{array}$ \\
\hline $\begin{array}{l}\text { Pan } \\
\text { Fire } \\
\text { Size } \\
\text { (m) } \\
\end{array}$ & $\begin{array}{c}\text { Time to } \\
\text { lst } \\
\text { Crack } \\
\text { (sec) }\end{array}$ & $\begin{array}{l}\text { Predicted } \\
\text { Time } \\
\text { (sec) }\end{array}$ & $\begin{array}{c}\text { Time to } \\
\text { lst } \\
\text { Crack } \\
\text { (sec) } \\
\end{array}$ & $\begin{array}{l}\text { Predicted } \\
\text { Time } \\
\text { (sec) }\end{array}$ & $\begin{array}{c}\text { Time to } \\
\text { 1st } \\
\text { Crack } \\
\text { (sec) } \\
\end{array}$ & $\begin{array}{l}\text { Predicted } \\
\text { Time } \\
\text { (sec) }\end{array}$ \\
\hline $\begin{array}{c}0.5 \times 0.5 \\
\text { corner }\end{array}$ & 347 & 212 & 578 & DNF & 326 & 207 \\
\hline $\begin{array}{c}0.7 \times 0.7 \\
\text { corner }\end{array}$ & 126 & 122 & 234 & DNF & 136 & 121 \\
\hline $\begin{array}{c}0.8 \times 0.8 \\
\text { corner }\end{array}$ & 131 & 111 & 202 & DNF & 121 & 109 \\
\hline $\begin{array}{c}0.9 \times 0.9 \\
\text { corner }\end{array}$ & 70 & 86 & 145 & DNF & 82 & 85 \\
\hline $\begin{array}{c}0.6 \times 0.6 \\
\text { centre }\end{array}$ & 475 & 316 & 675 & DNF & 857 & 307 \\
\hline $\begin{array}{c}0.7 \times 0.7 \\
\text { centre }\end{array}$ & 282 & 276 & 348 & DNF & 315 & 271 \\
\hline $\begin{array}{c}0.8 \times 0.8 \\
\text { centre }\end{array}$ & 195 & 196 & 309 & 206 & 111 & 193 \\
\hline $\begin{array}{c}0.9 \times 0.9 \\
\text { centre }\end{array}$ & 126 & 257 & 156 & 257 & 110 & 255 \\
\hline
\end{tabular}

DNF $=$ did not fracture

A breaking stress of $\sigma_{\mathrm{f}}=47 \mathrm{MPa}$ was used as before. BRANZFIRE (version 2002.6) was used to simulate the fire environment in the compartment and to predict the time of first 
fracture for each glazed pane. The heat release rate for each size of pan as published by Shields et al [21, 22] was used as input with fuel properties selected as for ethanol. The predicted glass fracture times are compared with the measured time to first cracking and results summarised in Table 5.

Best agreement was obtained for Pane 1 where the glass was entirely submerged in the hot layer. BRANZFIRE was not able to adequately predict the fracture time for Pane 2 for the corner fires where the glass was predicted to be located entirely within the lower gas layer in the room. In general the model shows a tendency to under predict the time of first fracture, but overall the results are considered reasonable given the modelling uncertainties and potential variability of glass fracture strength discussed elsewhere in this paper. The data presented in Table 5 apply to the specific experiments associated with the published heat release rate data. There were also duplicate experiments reported (typically 3-5) for each pan size and location. The data is presented graphically in Figure 6 with error bars indicating the uncertainty associated with the experimental data.

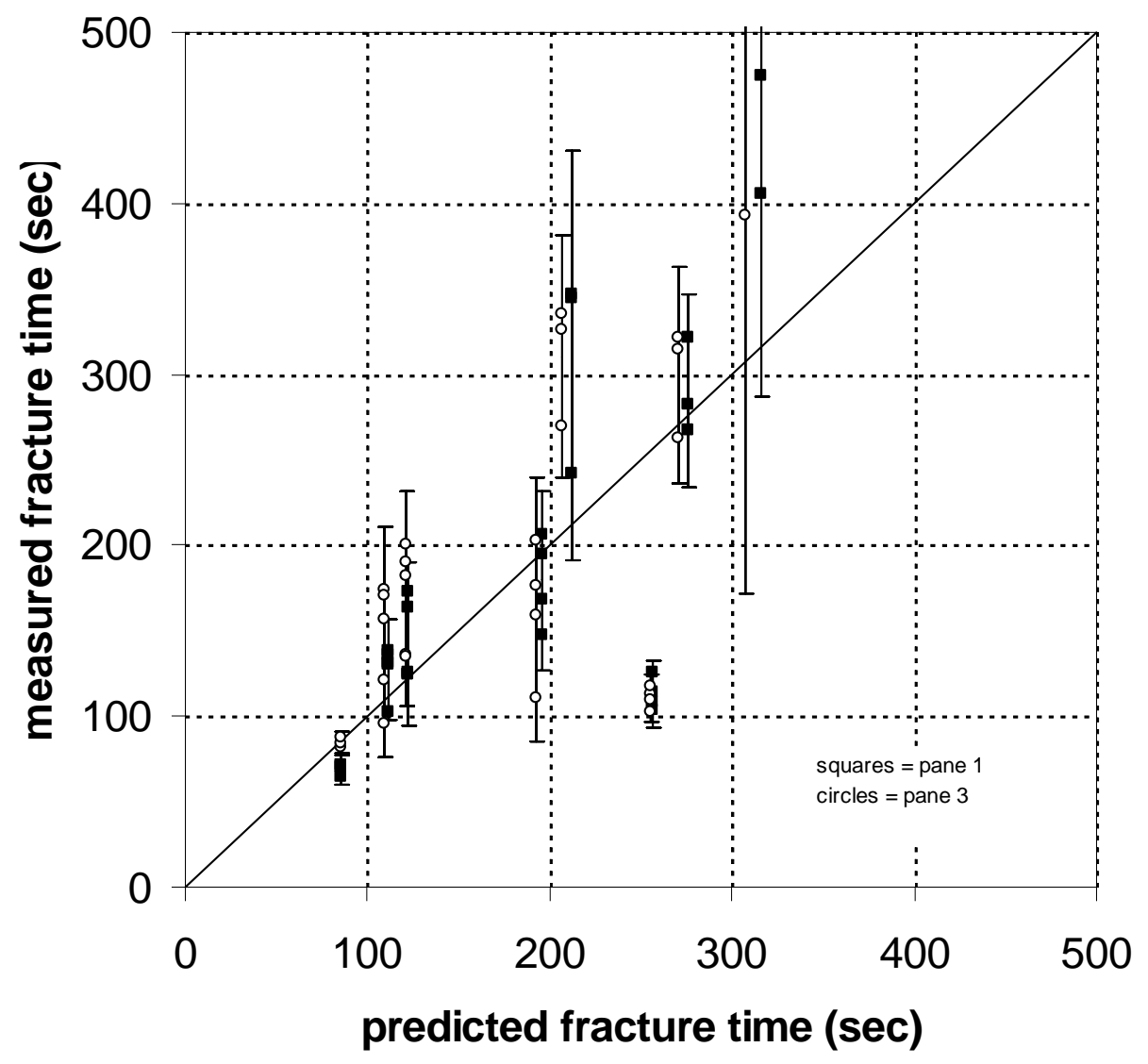

Figure 6 : Comparison of predicted vs measured glass fracture times for panes 1 and 3 (error bars span 2 standard deviation) 
Generally the predictions are within the experimental range of uncertainly except for the 900 mm square pan located in the centre of the room where the experimental fracture times were much shorter than the predicted values. It is likely that in this case direct radiation from the flame to the glass was significant and was not accounted for in the predicted value.

\section{SENSITIVITY ANALYSIS}

The glass fracture model was also implemented in a spreadsheet, allowing a reasonably thorough analysis of the sensitivity of the model to all significant input parameters. This analysis has been carried out using Microsoft Excel 2000 (version 9.0) and version 4.0.5 of the @Risk spreadsheet add-in [31]. Probability distributions are assigned to each input variable, and Monte Carlo analysis is used to generate a probability distribution for selected output variables. Tornado charts are also generated showing the most important input variables for a given output. Tornado charts are a form of bar chart with vertical category axes that provide a ranking of input variable sensitivities and often have a characteristic "tornado" shape. The spreadsheet used the compartment gas layer temperature taken from the BRANZFIRE output as given so that the sensitivity of the glass fracture time, to changes in the compartment gas temperature was not assessed as part of this exercise. However changes in heat release rate of the fire and compartment temperatures will naturally have a significant effect on the glass fracture times as shown comparing the fractures times from BRANZFIRE for the different assumed combustion efficiencies.

\section{Input and Output Variables}

Input variables and initial probability distributions are listed in Table 6. The mean window dimensions are the same as the simulations used for the BRANZFIRE - BREAK1 comparison. The sensitivity analysis was run with both medium and ultra-fast t-squared fire temperature histories. Generic distributions have been used for most variables, as there is little data available on actual distributions of the physical properties involved. 
Table 6 - Initial @Risk Input Variable Distributions

\begin{tabular}{|c|c|c|c|}
\hline Variable & Distribution & Parameters & Comments \\
\hline Shaded edge & Triangular & $\begin{array}{l}\text { Min }=13.5 \mathrm{~mm} \\
\text { Mean }=15 \mathrm{~mm} \\
\text { Max }=16.5 \mathrm{~mm}\end{array}$ & $\pm 10 \%$ \\
\hline Thickness & Triangular & $\begin{array}{l}5.7 \mathrm{~mm} \\
6.0 \mathrm{~mm} \\
6.3 \mathrm{~mm}\end{array}$ & $\pm 5 \%$ \\
\hline Half-width & Triangular & $\begin{array}{l}0.36 \mathrm{~m} \\
0.40 \mathrm{~m} \\
0.44 \mathrm{~m} \\
\end{array}$ & $\pm 10 \%$ \\
\hline Fracture Strength & $\begin{array}{l}\text { Weibull } \\
\text { (2 parameter) }\end{array}$ & $\begin{array}{l}\alpha=3.2 \\
\beta=74.1 \mathrm{MPa}\end{array}$ & J oshi and Pagni [8] \\
\hline Young's Modulus & Normal & $\begin{array}{l}\mu=72 \mathrm{MPa} \\
\sigma=7.2\end{array}$ & $\begin{array}{l}\text { Coefficient of Variation } \\
(\mathrm{CoV})=10 \%\end{array}$ \\
\hline $\begin{array}{l}\text { Thermal Expansion } \\
\text { Coefficient }\end{array}$ & Normal & $\begin{array}{l}\mu=9.5 \times 10^{-6} \mathrm{~K}^{-1} \\
\sigma=9.5 \times 10^{-7}\end{array}$ & $\mathrm{CoV}=10 \%$ \\
\hline Thermal Diffusivity & Normal & $\begin{array}{l}\mu=3.6 \times 10^{-7} \mathrm{~m}^{2} / \mathrm{s} \\
\sigma=3.6 \times 10^{-8}\end{array}$ & $\mathrm{CoV}=10 \%$ \\
\hline Thermal conductivity & Normal & $\begin{array}{l}\mu=0.76 \mathrm{~W} / \mathrm{mK} \\
\sigma=0.076\end{array}$ & $\mathrm{CoV}=10 \%$ \\
\hline Interior temperature & Triangular & 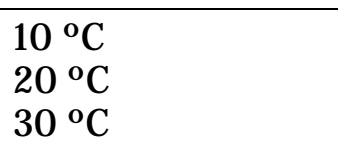 & \\
\hline Exterior temperature & Normal & $\begin{array}{l}\mu=15{ }^{\mathrm{o}} \mathrm{C} \\
\sigma=5{ }^{\circ} \mathrm{C}\end{array}$ & \\
\hline Exterior emissivity & Uniform & $\begin{array}{l}\operatorname{Min}=0 \\
\operatorname{Max}=1\end{array}$ & \\
\hline $\begin{array}{l}\text { Exterior convection } \\
\text { coefficient }\end{array}$ & Triangular & $\begin{array}{l}5 \mathrm{~W} / \mathrm{m}^{2} \mathrm{~K} \\
10 \mathrm{~W} / \mathrm{m}^{2} \mathrm{~K} \\
15 \mathrm{~W} / \mathrm{m}^{2} \mathrm{~K}\end{array}$ & $\pm 50 \%$ \\
\hline $\begin{array}{l}\text { Maximum interior } \\
\text { convective coefficient }\end{array}$ & Triangular & $\begin{array}{l}45 \mathrm{~W} / \mathrm{m}^{2} \mathrm{~K} \\
50 \mathrm{~W} / \mathrm{m}^{2} \mathrm{~K} \\
55 \mathrm{~W} / \mathrm{m}^{2} \mathrm{~K}\end{array}$ & $\pm 10 \%$ \\
\hline $\begin{array}{l}\text { Minimum interior } \\
\text { convective coefficient }\end{array}$ & Triangular & $\begin{array}{l}4.5 \mathrm{~W} / \mathrm{m}^{2} \mathrm{~K} \\
5.0 \mathrm{~W} / \mathrm{m}^{2} \mathrm{~K} \\
5.5 \mathrm{~W} / \mathrm{m}^{2} \mathrm{~K}\end{array}$ & $\pm 10 \%$ \\
\hline
\end{tabular}

The interior and exterior temperatures were correlated, with strength of 0.5 , indicating a moderate level of coincidence between high exterior and high interior temperatures. The simulations were run with the random number seed 3 and set to auto-stop on convergence. This allows @Risk to stop the simulation once key parameters of the output distributions have stabilised. The medium fire simulation stopped after 300 iterations, while the ultra-fast fire simulation stopped after 475 iterations. 


\section{Results}

The tornado charts for the critical temperature increase were essentially identical for both the ultra-fast and medium growth rate fires. The chart for the ultra-fast fire is shown in Figure 7. This shows that the fracture strength is by far the most important input variable affecting the fracture temperature. The fracture temperature is positively correlated with fracture strength, indicating that higher strength glass will have a higher fracture temperature. Young's modulus and thermal expansion coefficient are near equally important as second and third most significant variables. They are both negatively correlated with fracture temperature.

The relative importance of the input variables for the fracture time differs slightly between the ultra-fast and medium scenarios. The rankings and values are presented in Table 7. In both scenarios, the most significant variable is once again the fracture strength. The thermal expansion coefficient and Young's modulus of the glass are the next most significant inputs, followed by the thermal diffusivity and thermal conductivity of the glass. The generated probability distribution for the fracture time from the ultra-fast scenario ranges from 48 seconds to 365 seconds. The mean is 128 seconds and the standard deviation is 35 seconds. This compares to the standard spreadsheet output of 125 seconds using fracture strength of $47 \mathrm{MPa}$.

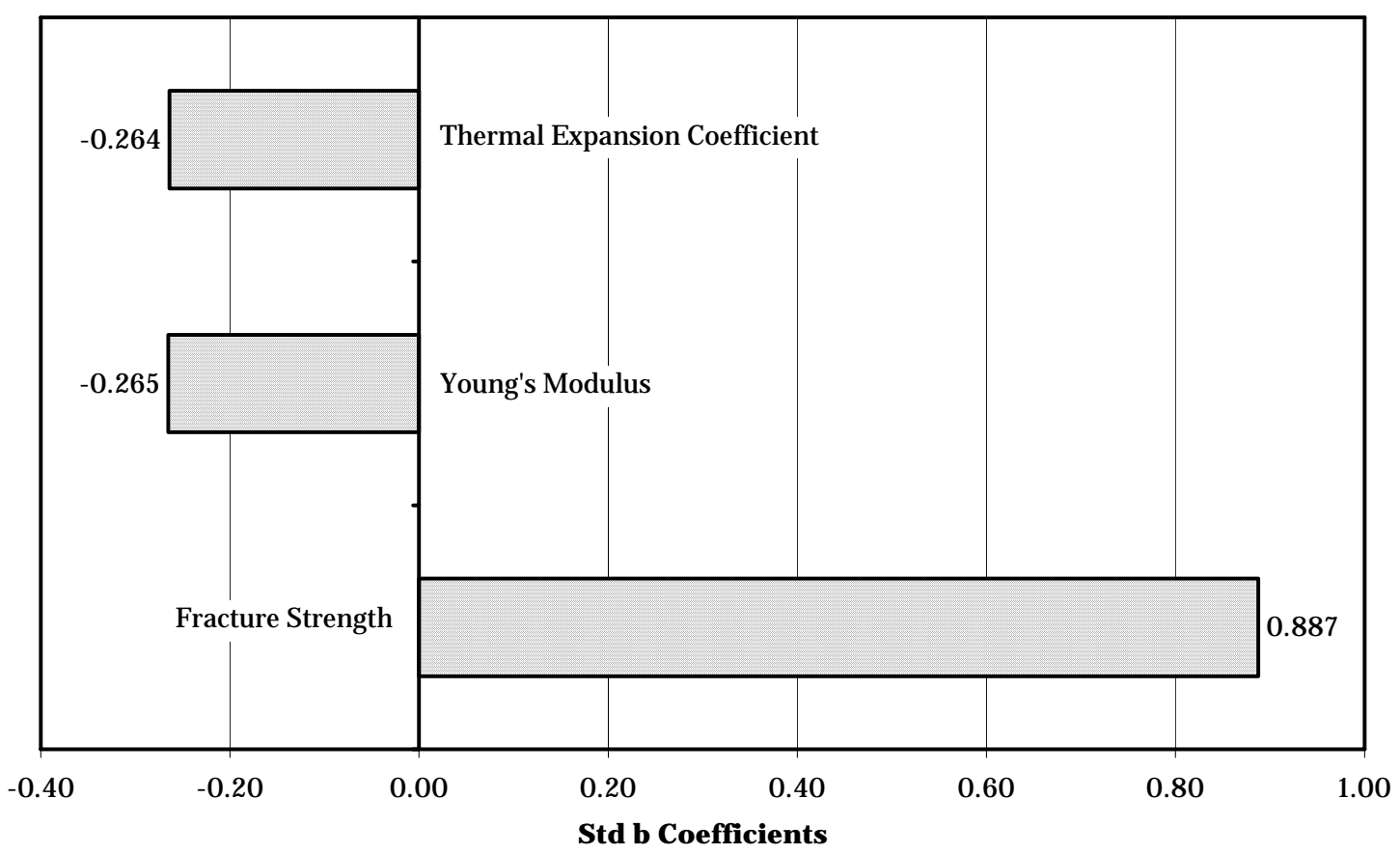

Figure 7 - Tornado chart: regression sensitivity of critical temperature rise 
Table 7 - Sensitivity ranking comparison for the fracture time

\begin{tabular}{|l|c|c|c|c|}
\hline Variable & $\begin{array}{c}\text { Ranking } \\
\text { (ultra-fast } \\
\text { growth fire) }\end{array}$ & Value & $\begin{array}{c}\text { Ranking } \\
\text { (medium } \\
\text { growth fire) }\end{array}$ & Value \\
\hline Fracture Strength & 1 & .825 & 1 & .773 \\
\hline $\begin{array}{l}\text { Thermal expansion } \\
\text { coefficient }\end{array}$ & 2 & -.272 & 3 & -.224 \\
\hline Young's modulus & 3 & -.256 & 2 & -.237 \\
\hline Thermal diffusivity & 4 & -.205 & 5 & -.104 \\
\hline Thermal conductivity & 5 & .198 & 4 & .117 \\
\hline $\begin{array}{l}\text { Maximum convection } \\
\text { coefficient }\end{array}$ & 6 & -.081 & 7 & -.064 \\
\hline Interior temperature & 7 & .039 & - & \\
\hline Glass thickness & 8 & .03 & 6 & .069 \\
\hline $\begin{array}{l}\text { Exterior convection } \\
\text { coefficient }\end{array}$ & - & - & 8 & .06 \\
\hline
\end{tabular}

\section{Discussion}

The relative significance of an input variable, as measured by @Risk, accounts for both functional dependence and statistical variation. An input variable with a broad probability distribution will generally show up as a significant variable, as will an input variable with a high functional dependence. The standard variation of the probability distribution for the fracture strength is an order of magnitude larger than that of any other input variable. It is largely this factor that leads the fracture strength to be the most significant input variable for both fracture strength and fracture time.

The five most significant variables for the fracture time are all physical properties of the glass. The next three variables have regression values so low that their rankings are inconclusive. The top three input variables are all properties that affect the fracture temperature. This indicates that it is the fracture stress model that has a greater uncertainty than the heat transfer model. This may be misleading, however, as the accuracy of the heat transfer model is dependent upon more assumptions regarding values such as heat transfer coefficients, radiative absorption coefficients, compartment gas temperature profiles and emissivity.

\section{CONCLUSIONS}

- The spreadsheet and BRANZFIRE implementations of the glass fracture model largely developed by Sincaglia and Barnett have been successful. This was demonstrated by 
successful verification against an alternative software model (BREAK1) and against experimental results.

- Improvement has been made to the finite difference scheme stability criterion derived by Sincaglia and Barnett.

- BRANZFIRE predicts fracture times in close agreement with Pagni and Joshi's BREAK1 for glass thicknesses of 3 and $6 \mathrm{~mm}$, and slightly quicker fracture times for $12 \mathrm{~mm}$ glass. This was shown using 12 simulations with a range of fires and window properties.

- BRANZFIRE predicted a fracture time consistent with experimental results from Skelly, Roby and Beyler.

- BRANZFIRE predicted fracture times in reasonable agreement with those measured by Shields et al, taking into account the uncertainty in the experimental data and glass properties. Best agreement was when the glazing was located entirely within the upper layer.

- Monte Carlo simulation of the spreadsheet input variables identified fracture strength as the most significant glass property variable affecting fracture time.

- The glass fracture model described herein has been shown to be based on proper physical assumptions and compares well with available experimental data; however readers are reminded that glass fracture has no direct effect on development of room fires. In contrast glass fallout will have a significant effect on ventilation and fire development. Fracture or cracking is a necessary but not sufficient condition for fallout to take place. Furthermore, significant fallout generally takes place some time later following initial cracking, and sometimes fallout does not happen at all. Further research is therefore essential to determine the physical basis for predicting glass fallout.

\section{ACKNOWLEDGEMENTS}

The authors acknowledge the New Zealand Fire Service Commission for their support of the fire engineering program at the University of Canterbury, and the Foundation for Research Science and Technology for support of fire modelling research at BRANZ. 


\section{NOMENCLATURE}

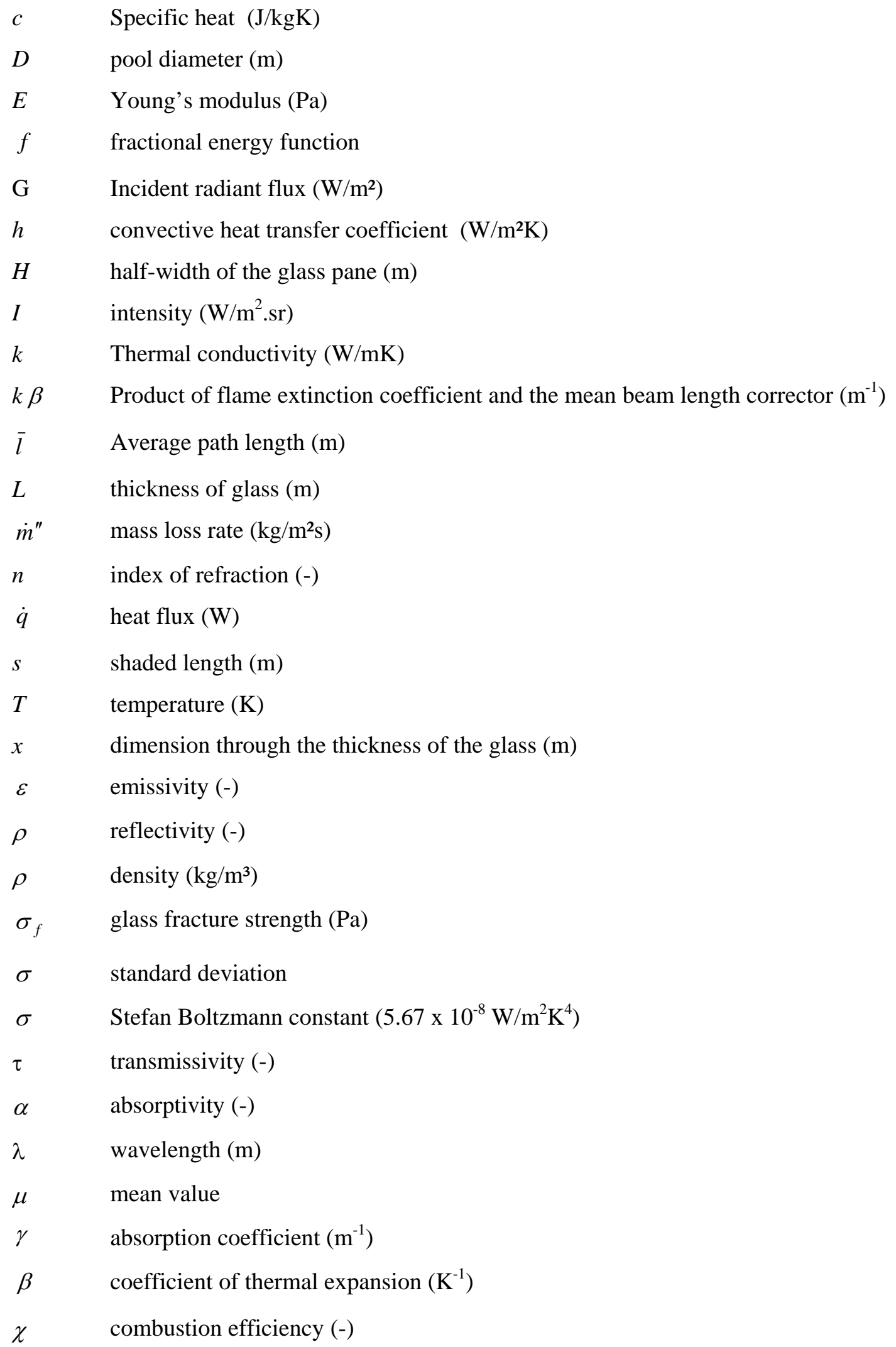


Subscripts

E Exterior side

I interior side

U upper layer

$i \quad$ at the $i$ th node

$o \quad$ initial value 


\section{REFERENCES}

1. Wade, C. A. BRANZFIRE Technical Reference Guide. BRANZ Study Report 92. Building Research Association of New Zealand, Judgeford, New Zealand. 2002.

2. Parry, R. Implementation of a Glass Fracture Module for the BRANZFIRE Compartment Fire Zone modelling Software. Master of Fire Engineering Research Project Report. Department of Civil Engineering, University of Canterbury, New Zealand. 2002.

3. Emmons, H. W. The Needed Fire Science. Fire Safety Science - Proceedings of the First International Symposium (Berkeley), 1985, pp 33-53.

4. Pagni, P. J. Fire Physics - Promises, Problems, and Progress. Fire Safety Science - Proceedings of the Second International Symposium (Tokyo), 1989, pp 49-66.

5. Pagni, P. J. and Joshi, A. A. Glass Breaking in Fires. Fire Safety Science - Proceedings of the Third International Symposium (Edinburgh), 1991, pp 791-802.

6. Joshi, A. A., and Pagni, P. J. Users' Guide to BREAK1, The Berkeley Algorithm for Breaking Window Glass in a Compartment Fire. National Institute of Standards and Technology, Gaithersbury, MD, 1991.

7. Joshi, A. A., and Pagni, P. J. Fire Induced Thermal Fields in Window Glass. I - Theory. Fire Safety Journal Vol. 22, 1994, pp 25-44.

8. Joshi, A. A., and Pagni, P. J. Fire Induced Thermal Fields in Window Glass. II - Experiments. Fire Safety Journal. Vol. 22, 1994, pp 45-65.

9. Keski-Rahkonen, O. Breaking of Window Glass Close to Fire. Fire and Materials, Vol. 12, 1988, pp 61-69.

10. Keski-Rahkonen, O. Breaking of Window Glass Close to Fire, II: Circular Panes. Fire and Materials, Vol. 15, No. 1, 1991, pp 11-16.

11. Cuzzillo, B. R., and Pagni, P. J. Thermal Breakage of Double-Pane Glazing by Fire. J. of Fire Prot. Engr. Vol. 9, No. 1, 1998, pp 1-11.

12. Sincaglia P. E. and Barnett, J. R. Development of a Glass Window Fracture Model for Zone-Type Computer Fire Codes. J. of Fire Prot. Engr., Vol. 8, No. 3, 1997, pp 101-118. 
13. Gardon, R. Calculation of Temperature Distributions in Glass Plates Undergoing Heat Treatment. Journal of the American Ceramic Society. Vol. 41, 1958, pp 200-208.

14. Skelly, M. J., Roby, R. J., and Beyler, C. L. An Experimental Investigation of Glass Breakage in Compartment Fires. J. of Fire Prot. Engr., Vol. 3, No. 1, 1991, pp 25-34.

15. Shields, T. J., Silcock, G. W. H., and Hassani, S. K. S. Behaviour of Glazing in a Large Simulated Office Block in a Multi-Story Building. J. Applied Fire Science. Vol. 7, No. 4, 1998, pp 333-352.

16. Hassani, S. K. S., Shields, T. J., Silcock, G. W. H. An Experimental Investigation into the Behaviour of Glazing in Enclosure Fire. In: The Behaviour of Glass and Other Materials Exposed to Fire: Volume I. Ed: P. R. DeCicco, Baywood Publishing Company, New York, pp 3-22, 2002.

17. Hassani, S. K. S., Shields, T. J., Silcock, G. W. H. In Situ Experimental Thermal Stress Measurements in Glass Subjected to Enclosure Fires. In: The Behaviour of Glass and Other Materials Exposed to Fire: Volume I. Ed: P. R. DeCicco, Baywood Publishing Company, New York, pp 39-50, 2002.

18. Shields, T. J., Silcock, G. W. H., Hassani, S. K. S. The Behaviour of Single Glazing in an Enclosure Fire. In: The Behaviour of Glass and Other Materials Exposed to Fire: Volume I. Ed: P. R. DeCicco, Baywood Publishing Company, New York, pp 51-69, 2002.

19. Shields, T. J., Silcock, G. W. H., Hassani, S. K. S. The Behaviour of Double Glazing in an Enclosure Fire. In: The Behaviour of Glass and Other Materials Exposed to Fire: Volume I. Ed: P. R. DeCicco, Baywood Publishing Company, New York, pp 71-89, 2002.

20 Harada, K., Enomoto, A., Uede, K., Wakamatsu, T. An Experimental Study on Glass Cracking and Fallout by Radiant Heat Exposure. Fire Safety Science - Proceedings of the Sixth International Symposium (France), 1999, pp 1063-1074.

21. Shields, T.J., Silcock, G.W.H., Flood, M. Performance of a Single Glazing Assembly Exposed to a Fire in the Centre of an Enclosure. Fire and Materials. Vol 26, pp51-75, 2002.

22. Shields, T.J., Silcock, G.W.H., Flood, M. Performance of a Single Glazing Assembly Exposed to Enclosure Corner Fires of Increasing Severity. Fire and Materials. Vol 25, pp123-152, 2002. 
23. Hassani, S. K. S., Shields, T. J., Silcock, G. W. H. Thermal Fracture of Window Glazing: Performance of Glazing in Fire. In: The Behaviour of Glass and Other Materials Exposed to Fire: Volume I. Ed: P. R. DeCicco, Baywood Publishing Company, New York, pp 23-37, 2002.

24. Mai, Y. W., and Jacob, L. J. S. Thermal Stress Fracture of Solar Control Window Panes Caused by Shading of Incident Radiation. Matériaux et Constructions. Vol. 13, No. 76, pp 283-288, 1980.

25. Babrauskas, V. Glass Breakage in Fires. World Wide Web, http://www.interfire.org/features/glass_breakage.htm [accessed 12/2001]. 1997.

26. Incropera, F. P. and DeWitt, D. P. Fundamentals of Heat and Mass Transfer. Fourth Edition. John Wiley \& Sons, New York, 1996.

27. Siegel, R. and Howell, J. R. Thermal Radiation Heat Transfer. Third Edition. Hemisphere Publishing Corporation, Washington, 1992.

28. Babrauskas, V. Burning Rates. In: The SFPE Handbook of Fire Protection Engineering. Second Edition. National Fire Protection Association, Quincy, MA, 1995.

29. Tewarson, A. Generation of Heat and Chemical Compounds in Fires. In: The SFPE Handbook of Fire Protection Engineering. Second Edition. National Fire Protection Association, Quincy, MA, 1995.

30. Pilkington North America Inc. Technical Information Properties of Soda-Lime-Silica Float Glass. ATS-129. 6 March 2002.

31. Palisade Corporation. Guide to Using @Risk - Risk Analysis and Simulation Add-in for Microsoft Excel. Version 4. NY, USA. March 2000. 